\title{
Introduction to the special issue: Languages and software in robotics*
}

\author{
J.A. Rose (Guest Editor)
}

School of Computing, Staffordshire University, Box 334, Beaconside, Stafford ST18 ODG (U.K.)

Section A of the special issue consisting of 13 papers covering a wide field is devoted to the topic of languages and software in robotics, with an emphasis on practical applications. Diverse themes are analysed and discussed, such as neural networks, fuzzy approaches, objectoriented programming, simulation and modelling, graphical robot languages and other pertinent topics. Authors from a number of countries have contributed papers describing recent research and different approaches to this important field, particularly in view of the potential applications in various areas.

The first three papers deal with neural network control. Mao and Hsia discuss the obstacle avoidance inverse kinematics solution of redundant robots by neural networks. Training algorithms are developed and the technique used is computationally efficient. The second paper by Cha and Choo develops a neurofuzzy approach for the control of telerobot systems that can work in an on-line manner, as verified in laboratory experiments. The third paper in this section by Jezernik et al. discusses neural network control design with sliding modes to improve the robustness in learning control; this method was found to be effective in experimental work, even in non-linear robot systems.

The fourth paper by Shiller is devoted to work-cell layout design and optimal robot motion planning. It describes an innovative approach to off-line robot programming, and the graphic displays provide a useful tool for interactive motion planning and work-cell design.

The next paper by Zielinski presents an objectoriented approach to the implementation of a software library which contains building blocks for the construction of multi-robot system controllers for specific tasks. The authors provides a valuable survey of various programming methods for robots, e.g. on-line and off-line programming, hybrid programming and openstructure programming systems.

Another approach for programming complex robotic systems is outlined in the paper by $\mathrm{Ma}$ et al., who describe a generic development and simulation facility for such systems. This work formed part of Canada's contribution to the International Space Station program. The authors supply examples of current applications of program.

The seventh paper by Zeghloul et al. describes a robot modelling and simulation system which allows the user to

* Section B of the special issues will appear in vol. 15 part 2 (March-April, 1997). deal with a large number of problems in robotics. A graphic simulator is used to resolve problems arising in CAD-robotics systems, e.g. collision free-path planning.

The problem of a task planning language is considered in the eighth paper by Kuo and Sanger. It is assumed that the manipulator is an elastic systems; this permits the development of a task planning method by using analyses that are mutually coherent. The method is claimed to enable a manipulator to simultaneously avoid obstacles, singularities and joint motion limitations. The task planning method applies to the general serial redundant manipulator with an arbitrary degree of redundancy.

In the following paper by Liu and Qin examine the situation involving an unknown environment. To solve this difficult problem the authors developed an integrated software bench, called $C^{4}$ for modelling and simulating autonomous robotic systems, e.g. legged and dual-arm manipulators. This enables the device to self-organise its own behaviour in order to adapt to an unknown environment. The technique also applies to interactions between individual robots in a complex system.

The final four papers deal with diverse topics relating to practical applications. The first in this group (paper No. 10) is a graphical robot language developed by Arai et al. in Japan, as a part of a three year programme still in operation; it describes in detail a graphical robot programming system that is user-friendly, and it is designed for programming an arc welding robot in small batch production where low-skill workers are employed.

The second paper in this group of 4 relates to a syntax-directed method in which the syntax of the input of the application plays a central role. Based on the method the author, A. Hoohewijs, developed a description, termed LL(1), that can be used to produce automatically motion control softwarer, object recognizers and human-computer interfaces.

A programming environment for robotic systems, termed ORCCAD, is dealt with in the third paper of the group. According to its authors, D. Simon et al., ORCCAD gathers sontrol laws in continuous time at the low levels and in discrete time at higher levels. Complex applications can then be designed, verified and generated incrementally.

Section B of the special issue will appear in the next part of volume 15 (March-April, 1997) and will comprise papers concerned with unmodelled and manipulation environments, as well as will languages used in 
rehabilitation robotics. In view of the exciting new developments in the pipeline concerning man-machine interactions, it is hoped to publish in about three years time another special issue devoted to languages and software in robotics, a rapidly expanding and challenging field of research work and practical applications.

Finally, I would like to thank the contributors for their goodwill and patience, and the Publishers, Printers and the editor, as well as the referees, for their help. 